\title{
Distributed Auxiliary Particle Filtering with Diffusion Strategy for Target Tracking: A Dynamic Event-Triggered Approach
}

\author{
Weihao Song, Zidong Wang, Jianan Wang, Fuad E. Alsaadi and Jiayuan Shan
}

\begin{abstract}
This paper investigates the particle filtering problem for a class of nonlinear/non-Gaussian systems under the dynamic event-triggered protocol. In order to avert frequent data transmission and reduce the communication overhead, a dynamic event-triggered transmission mechanism is adopted to decide whether the data should be transmitted or not. We first consider a scenario where all sensor nodes selectively transmit their newly obtained measurements to a central node, and a full likelihood function at the central node is derived by fusing the transmitted measurements and the information embodied in the non-triggered measurements. Based on the derived full likelihood function, a centralized auxiliary particle filtering algorithm is proposed to select those particles that are more likely to match the current measurement information. Next, based on the diffusion strategy, a distributed auxiliary particle filtering algorithm is further developed, where the local measurements and the local posteriors (approximated by the Gaussian mixture models) are exchanged among neighboring nodes under the dynamic eventtriggered communication strategy. Finally, the effectiveness of the proposed filtering schemes is demonstrated via Monte Carlo simulations in a target tracking problem with received-signalstrength sensors.
\end{abstract}

Index Terms-Distributed particle filtering, auxiliary particle filtering, dynamic event-triggered mechanism, diffusion strategy, nonlinear/non-Gaussian systems

\section{INTRODUCTION}

Over the past few decades, the target tracking problem has been of great significance in both military and civilian applications with examples including ballistic missile tracking [53], mobile phone user tracking [59], and the speaker tracking [60]. A crucially important task of the target tracking problems is the state estimation whose primary purpose is to make statistical inference about the state of the target by using the incoming measurements (e.g., range and bearing) obtained from sensors [12], [30], [32], [38], [51]. As a popular state estimation approach, the Bayes filtering algorithm aims to

The Deanship of Scientific Research (DSR) at King Abdulaziz University, Jeddah, Saudi Arabia funded this project, under grant no. (FP-24-42). This work was also supported in part by the National Natural Science Foundation of China under Grant 61873031, 61873148, and 61933007, the China Scholarship Council under Grant 201806030206, and the Alexander von Humboldt Foundation of Germany. (Corresponding author: Jianan Wang.)

W. Song, J. Wang and J. Shan are with the School of Aerospace Engineering, Beijing Institute of Technology, Beijing 100081, China. (Email: wangjianan@bit.edu.cn)

Z. Wang is with the Department of Computer Science, Brunel University London, Uxbridge, Middlesex, UB8 3PH, United Kingdom. (Email: Zidong.Wang@brunel.ac.uk)

F. E. Alsaadi is with the Department of Electrical and Computer Engineering, Faculty of Engineering, King Abdulaziz University, Jeddah 21589, Saudi Arabia. obtain the state estimate by means of recursively updated posterior probability density function (PDF) of the target's state [26].

For the Bayes filtering method, the integrations over the state space are involved in the recursive propagation, which renders substantial difficulties in deriving the closed-form expression for the posterior PDF. An exception that needs to be mentioned is the linear systems with additive Gaussian noises, where the posterior PDF is Gaussian and its parameters (i.e. mean and covariance) can be updated via the well-known Kalman filtering scheme [39]. To deal with the filtering problem for the frequently encountered nonlinear/nonGaussian systems in practical applications, a variety of filtering approaches have been developed based on different approximation techniques. Among others, we mention extended Kalman filtering [37], cubature Kalman filtering [2], unscented Kalman filtering [28], and sparse-grid quadrature filtering approaches [27]. Apart from these Gaussian-assumption-based filtering methods, the Monte-Carlo-based particle filtering algorithm [3], [36], [58] has recently attracted ever-increasing attention due mainly to its distinctive capability of handling nonlinearity/non-Gaussianity.

With the continuous revolution of smart sensor and wireless communication technologies, it becomes a rather common practice to detect and track a target of interest by a large number of sensor nodes capable of sensing, communicating, storing and processing data [57]. When it comes to the state estimation problems, the main idea of the traditional centralized approach is that all local sensor nodes transmit their raw or quantized measurements to a central node and then the central node processes all the measurements simultaneously to obtain a global estimate, see e.g., [13], [14]. Clearly, with such a centralized approach, the entire system is prone to fault/failure of the central node and/or the unbearable computational burden. As such, a more preferable approach by industry is to develop distributed algorithms without having to rely on the central node, where each sensor node only needs to communicate with its neighboring nodes [45], [50].

In the past few decades, the distributed state estimation problems have drawn a recurring research interest with a great many results available in the literature [5], [8], [13], [15], [48]. For example, a distributed particle filtering algorithm has been developed in [15] by using the parametric model to approximate the global likelihood function, and another algorithm has also been proposed based on the quantized measurements to reduce the communication overhead. In [48], 
This article has been accepted for publication in a future issue of this journal, but has not been fully edited. Content may change prior to final publication. Citation information: DOI10.1109/TSP.2020.3042947, IEEE Transactions on Signal Processing

a distributed particle filter has been developed for target tracking on the basis of the distributed resampling with nonproportional allocation method, which is able to conduct the resampling step in a parallelized way. From the perspective of parallel implementation, the total set of the particles has been divided into sub-sets (referred to as islands) in [56], and similarly, a cooperative filtering algorithm has been proposed in [43] to simultaneously achieve the state estimation and model selection, where a series of particle filters have been used to match the candidate models and each particle filter has been allocated a portion of the total number of particles.

Roughly speaking, there have been two mainstream schemes in the context of distributed state estimation, namely, the consensus-based scheme [5], [21], [34], [44] and diffusionbased scheme [11], [24]. For the former, a kind of distributed particle filtering algorithm has been proposed in [23], where each local particle filter uses the global likelihood function (calculated in a distributed manner based on likelihood consensus) to update the importance weights. In [20], the local likelihood function has been approximated by a Gaussian distribution, and only the mean and covariance have been propagated among the adjacent sensor nodes to calculate the global likelihood. In [8], the distributed particle filtering algorithms based on the quantization schemes have been proposed to reach a consensus on weights. Note that the consensus-based schemes ensure the consensus via iterative internode communication between two successive updates, while the diffusion-based ones are capable of processing the measurements in a more efficient way [24] and outperform the former over adaptive networks [55]. Accordingly, the diffusion-based state estimation problems have been paid considerable research effort in recent years. For example, a diffusion-based distributed interacting multiple model Kalman filtering algorithm has been proposed in [33] for Markov jump linear systems. In [10], a random exchange diffusion particle filtering algorithm has been developed and further extended to the case with unknown sensor model parameters. Furthermore, in [17], some light has been shed on the Bayesian explanation of diffusion estimation for a class of models with exponential family of distributions.

It is worth noting that, in most available results concerning distributed particle filtering problems, the periodic communication mechanism has been adopted under which the data transmission is conducted with a fixed interval, see the survey [22], [61] and the references therein. Such a timetriggered communication scheme often leads to unnecessary data transmissions, and is therefore unsuitable for wireless sensor networks with limited communication capacity, where the most energy-consuming operation is known to be the data transmission [4], [7], [52]. In search of an alternative communication mechanism that saves limited resources, the event-triggered communication mechanism (ETCM) has emerged as an excellent candidate which aims to schedule data transmissions, thereby reducing the resource consumption [6], [16], [54].

In the context of event-triggered mechanisms, the static ETCM uses a fixed triggering threshold regardless of the degree of necessity of event-triggering. Therefore, the static
ETCM is still conservative in reducing unnecessarily frequent data transmissions, and this situation has led to the emerging research interest on the dynamic ETCM whose threshold parameters are adjusted dynamically through the medium of a dynamic auxiliary variable, thereby further improving the resource utilization. Up to now, the dynamic ETCM has started to gain some initial attention with respect to the distributed state estimation problems for linear systems or nonlinear systems with special nonlinearities [19], [31]. Nevertheless, the corresponding ETCM-based distributed state estimation problem has not been adequately addressed yet for general nonlinear/non-Gaussian systems, and this constitutes the main motivation of our current investigation.

Based on the above discussions, a natural yet fundamental research topic is to develop a distributed particle filtering scheme for nonlinear/non-Gaussian systems under the dynamic ETCM, which is deemed to be a fairly challenging topic with the following essential difficulties: 1) how to develop the dynamic event-triggered particle filters where the dynamic ETCM is dedicatedly integrated for energy-saving purposes? 2) how to examine the effect from the dynamic ETCM on the performance of the proposed filtering schemes? and 3) how to exploit the information from the non-triggered measurements to assist in selecting the candidate particles and updating weights? In the current study, we are set to answer the abovementioned questions.

In this paper, we investigate the distributed auxiliary particle filtering problem for a class of general nonlinear/nonGaussian systems with the dynamic ETCM under which the data transmissions are governed in a dynamical way. The main contributions of this paper can be highlighted as follows.

1) A distributed implementation of the auxiliary particle filter based on the diffusion strategy is proposed for a class of general discrete-time nonlinear/non-Gaussian systems.

2) The dynamic event-triggered mechanism is introduced in the framework of particle filtering to reduce the frequency of data transmission and save energy consumption, which is more effective than the static counterpart.

3) The information from the non-triggered measurements is fully taken into consideration to help select the most promising particles and update the importance weights.

The remainder of this paper is outlined as follows. In Section II, the filtering issue is formulated for a class of nonlinear/non-Gaussian systems under the dynamic ETCM and the auxiliary particle filtering algorithm is briefly reviewed. In Section III, the centralized auxiliary particle filtering algorithm is firstly designed (where the transmissions of measurements are scheduled by the dynamic ETCM), and then extended to the distributed setting via the diffusion strategy. A practical application to the target tracking problem with received-signal-strength sensors is provided in Section IV to show the effectiveness of the proposed algorithms. Finally, some concluding remarks are drawn in Section V.

Notation. The notation used in this paper is fairly standard. $\mathbb{R}^{n}$ denotes the $n$-dimensional Euclidean vector space. The superscript $T$ represents the operation of transpose. $p_{x}(\cdot)$ stands for the PDF of a random variable $x$, i.e. $x \sim p_{x}(\cdot)$. $\mathbb{N}(x ; \mu, \Sigma)$ represents the Gaussian PDF of random variable 
This article has been accepted for publication in a future issue of this journal, but has not been fully edited. Content may change prior to final publication. Citation information: DOI10.1109/TSP.2020.3042947, IEEE Transactions on Signal Processing

FINAL VERSION

$x$ with mean $\mu$ and covariance $\Sigma$. $\mathbb{E}(x \mid y)$ stands for the mathematical expectation of $x$ conditional on $y$. $\|x\|_{W} \triangleq$ $\left(x^{T} W x\right)^{1 / 2}$ refers to the weighted norm of vector $x . x_{i: j}$ denotes the trajectory of $x$ from time instant $i$ to time instant $j$. Other notations will be given as required.

\section{Problem Formulation}

Consider the following discrete-time nonlinear system

$$
x_{k+1}=f\left(x_{k}\right)+\omega_{k},
$$

which is observed by a wireless sensor network comprising $N$ sensor nodes described by

$$
y_{k}^{i}=h^{i}\left(x_{k}\right)+\nu_{k}^{i}, \quad i=1,2, \ldots, N
$$

where $x_{k} \in \mathbb{R}^{n}$ and $y_{k}^{i} \in \mathbb{R}^{m}$ are, respectively, the state of the target plant and the measurement output of the $i$ th sensor node at time instant $k \cdot f(\cdot): \mathbb{R}^{n} \mapsto \mathbb{R}^{n}$ and $h^{i}(\cdot): \mathbb{R}^{n} \mapsto \mathbb{R}^{m}$ denote, respectively, the state transition function and the measurement function of the $i$ th sensor node. $\omega_{k} \in \mathbb{R}^{n}$ represents the process noise satisfying $p_{\omega_{k}}(\cdot)$ and $\nu_{k}^{i} \in \mathbb{R}^{m}$ is the measurement noise on the $i$ th sensor node satisfying $p_{\nu_{k}^{i}}(\cdot)$.

The following three assumptions are made to further clarify the considered system.

Assumption 1: The knowledge about the initial state $x_{0}$ is summarized by a known prior density $p_{x_{0}}(\cdot)$.

Assumption 2: The process noise $\omega_{k}$ and the measurement noises $\left\{\nu_{k}^{i}\right\}_{i=1}^{N}$ are mutually independent and also independent of the initial state $x_{0}$.

Assumption 3: The nonlinear functions $f(\cdot)$ and $h^{i}(\cdot)$ as well as the PDFs $p_{\omega_{k}}(\cdot)$ and $p_{\nu_{k}^{i}}(\cdot)$ are all known.

Remark 1: It is noteworthy that Assumption 3 has been widely adopted in a large body of literature regarding the recursive state estimation problems, not only for the particlefilter-related problems (see e.g., [3], [35], [62]), but for the Kalman-filter-related problems (see e.g., [27], [33]). In practice, the statistical properties of noises can be learned from historical data or the simulation experiments. Recently, there have been some reported results whose focuses are on developing the adaptive filters to deal with the problems with unknown or partially unknown noise parameters using the variational Bayesian approximation [49], which would be one of the possible extensions of the current investigation.

\section{A. Dynamic event-triggered communication mechanism}

Under the dynamic ETCM, the measurement output of the sensor node $i$ is transmitted to the central node or its neighboring nodes only when the pre-defined triggering condition is satisfied. The event generator function $g\left(r_{k}^{i}, \varrho_{k}^{i}, \theta_{k}^{i}\right)$ on sensor node $i$ is defined as [40]:

$$
g\left(r_{k}^{i}, \varrho_{k}^{i}, \theta^{i}\right)=\left\|r_{k}^{i}\right\|_{S^{i}}^{2}-\sigma^{i}-\frac{1}{\theta^{i}} \varrho_{k}^{i}
$$

where $r_{k}^{i}=y_{k}^{i}-\bar{y}_{k}^{i}$ denotes the difference between the current measurement $y_{k}^{i}$ and the latest transmitted measurement $\bar{y}_{k}^{i}$ of the $i$ th sensor node, $\sigma^{i}$ is a given positive scalar representing the triggering threshold, $S^{i}$ is a known weighting matrix and $\varrho_{k}^{i}$ is an auxiliary variable that evolves as

$$
\varrho_{k}^{i}=\chi^{i} \varrho_{k-1}^{i}-\left\|r_{k-1}^{i}\right\|_{S^{i}}^{2}+\sigma^{i}
$$

where $\theta^{i}$ and $\chi^{i}$ are both given parameters and $\varrho_{0}^{i}>0$ is the initial value.

The data transmission is executed if and only if the condition $g\left(r_{k}^{i}, \varrho_{k}^{i}, \theta_{k}^{i}\right)>0$ is satisfied. Before proceeding further, to describe whether or not the sensor node $i$ sends its current measurement to the fusion center or its neighboring nodes, an indicator variable $\gamma_{k}^{i}$ is introduced as follows:

$$
\gamma_{k}^{i}=\left\{\begin{array}{l}
1, \quad g\left(r_{k}^{i}, \varrho_{k}^{i}, \theta_{k}^{i}\right)>0 \\
0, \quad g\left(r_{k}^{i}, \varrho_{k}^{i}, \theta_{k}^{i}\right) \leq 0
\end{array}\right.
$$

Then, the actually available measurement information $z_{k}^{i}$ can be denoted as

$$
\left\{\begin{array}{l}
z_{k}^{i}=y_{k}^{i}, \quad \text { when } \gamma_{k}^{i}=1 \\
z_{k}^{i} \in \mathcal{Y}_{k}^{i}, \quad \text { when } \gamma_{k}^{i}=0
\end{array}\right.
$$

where $\mathcal{Y}_{k}^{i}=\left\{y_{k}^{i} \mid g\left(r_{k}^{i}, \varrho_{k}^{i}, \theta_{k}^{i}\right) \leq 0\right\}$.

Remark 2: For the dynamic ETCM described by (3)-(5), if the parameters $\theta^{i}$ and $\chi^{i}$ are selected as $\theta^{i} \geq \frac{1}{\chi^{i}}$ and $0<\chi^{i}<1$, then the auxiliary variable $\varrho_{k}^{i}$ is always nonnegative [19]. Consequently, the equivalent triggering threshold under the dynamic ETCM (i.e., $\sigma^{i}+\frac{1}{\theta^{i}} \varrho_{k}^{i}$ ) will never be less than that in the static counterpart (i.e., $\sigma^{i}$ ). In other words, the triggering times under the dynamic ETCM will be reduced and the communication burden will be relaxed. It should be noted that the equivalent triggering threshold decreases with the increase of the value of $\theta^{i}$. If $\theta^{i} \rightarrow \infty$, the dynamic event generator function (3) degenerates to $g\left(r_{k}^{i}\right)=\left\|r_{k}^{i}\right\|_{S^{i}}^{2}-\sigma^{i}$, which corresponds to the traditional static event-triggered mechanism. Furthermore, it is worthwhile to mention that the auxiliary variable $\varrho_{k}^{i}$ can be adjusted in real time using the measurement information. In practice, considering the limited network bandwidth and energy resource, the thresholdrelated parameters $\theta^{i}, \chi^{i}$ and $\sigma^{i}$ are preset in advance by the designer or engineer to cater for the real-world engineering specifications.

\section{B. Auxiliary particle filtering}

Given the measurements $z_{1: k}$, the minimum mean-square error (MMSE) estimate of the state $x_{k}$, denoted by $\hat{x}_{k}$, is defined as

$$
\hat{x}_{k}=\mathbb{E}\left\{x_{k} \mid z_{1: k}\right\}=\int x_{k} p\left(x_{k} \mid z_{1: k}\right) d x_{k} .
$$

Generally speaking, the closed form of the posterior $p\left(x_{k} \mid z_{1: k}\right)$ is difficult to obtain due to the existence of multidimensional integrals in the Bayesian recursion. A numerically efficient method is to approximate the posterior $p\left(x_{k} \mid z_{1: k}\right)$ by 
This article has been accepted for publication in a future issue of this journal, but has not been fully edited. Content may change prior to final publication. Citation information: DOI10.1109/TSP.2020.3042947, IEEE Transactions on Signal Processing

FINAL VERSION

a set of weighted particles $\left\{x_{k}^{m}, W_{k}^{m}\right\}_{m=1}^{M}$, and then we have

$$
\begin{aligned}
\hat{x}_{k} & =\int x_{k} p\left(x_{k} \mid z_{1: k}\right) d x_{k} \\
& =\int x_{k} \sum_{m=1}^{M} W_{k}^{m} \delta\left(x_{k}-x_{k}^{m}\right) d x_{k} \\
& =\sum_{m=1}^{M} W_{k}^{m} x_{k}^{m}
\end{aligned}
$$

where $\delta(\cdot)$ is the Dirac delta function, $x_{k}^{m}$ is drawn from a proposal distribution $q\left(x_{k} \mid x_{k-1}^{m}, z_{k}\right)$ and its corresponding weight $W_{k}^{m}$ is calculated by

$$
W_{k}^{m}=W_{k-1}^{m} \frac{p\left(z_{k} \mid x_{k}^{m}\right) p\left(x_{k}^{m} \mid x_{k-1}^{m}\right)}{q\left(x_{k}^{m} \mid x_{k-1}^{m}, z_{k}\right)} .
$$

Note that the importance weights need to be normalized, i.e., $W_{k}^{m}$ is reassigned according to $W_{k}^{m} / \sum_{n=1}^{M} W_{k}^{n}$.

A widely used proposal distribution is $q\left(x_{k} \mid x_{k-1}^{m}, z_{k}\right)=$ $p\left(x_{k} \mid x_{k-1}^{m}\right)$ which corresponds to the bootstrap particle filter. It should be noted that the current measurement information is not considered in the sampling stage, which may lead to certain performance degradation. To deal with such an issue, the so-called auxiliary particle filter is put forward in [47] by incorporating the knowledge of the newly obtained measurement before the sampling stage. This additional operation can increase the compatibility of the sampled particles with respect to the newly obtained measurement.

The main steps of the standard auxiliary particle filter are summarized as follows:

1) obtain the indices ind $d_{m}$ according to the probabilities proportional to $W_{k-1}^{m} p\left(z_{k} \mid \lambda_{k}^{m}\right)$, where $\lambda_{k}^{m} \sim$ $p\left(x_{k} \mid x_{k-1}^{m}\right)$

2) draw the new particle $x_{k}^{m}$ from $p\left(x_{k} \mid x_{k-1}^{i n d_{m}}\right)$;

3) calculate the corresponding weight according to

$$
W_{k}^{m}=\frac{p\left(z_{k} \mid x_{k}^{m}\right)}{p\left(z_{k} \mid \lambda_{k}^{i n d_{m}}\right)} .
$$

The purpose of this paper is to develop a distributed auxiliary particle filtering algorithm based on the diffusion strategy for the general nonlinear/non-Gaussian systems under the dynamic ETCM.

\section{Algorithm DeSIGN AND Discussion}

In this section, to facilitate the presentation, a central particle filtering algorithm under the dynamic ETCM is first derived. We assume that the transmission channels in the considered system are perfect, that is, the only cause for untransmitted/unreceived measurements is the failure to meet the triggering condition. Meanwhile, we also assume that the central node has the knowledge of the triggering condition on each sensor node. Immediately after the development of the centralized algorithm where the central node needs to simultaneously process all the measurements, a diffusion-based distributed implementation is proposed that only requires the internode communication within the local neighborhoods.

\section{A. Centralized auxiliary particle filtering under the dynamic event-triggered mechanism}

In the centralized filtering framework, all sensor nodes should send their observations to a central node to infer the posterior PDF. However, due to the effect of the dynamic ETCM, each sensor node will transmit its current observation to the central node only when the corresponding triggering condition is satisfied.

Let us define an indicator set $\Gamma_{k}=\left\{\gamma_{k}^{i}\right\}_{i=1}^{N}$, where $\gamma_{k}^{i}$ is introduced in (5), and use $\mathcal{I}_{k}$ to denote the set of the sensor nodes that transmit their current measurements to the central node at time instant $k$. It is worthwhile to point out that $\gamma_{k}^{i}$ is known in the filtering process. Motivated by [62], bearing in mind the fact that all the local measurements are independent given the state $x_{k}$, the full likelihood function for the central node is described as

$$
\begin{aligned}
& p\left(\Gamma_{k},\left\{y_{k}^{i}\right\}_{i \in \mathcal{I}_{k}} \mid x_{k}\right) \\
= & \int p\left(\Gamma_{k},\left\{y_{k}^{i}\right\}_{i \in \mathcal{I}_{k}},\left\{y_{k}^{i}\right\}_{i \in N \backslash \mathcal{I}_{k}} \mid x_{k}\right) d\left\{y_{k}^{i}\right\}_{i \in N \backslash \mathcal{I}_{k}} \\
= & \int p\left(\Gamma_{k} \mid x_{k},\left\{y_{k}^{i}\right\}_{i \in N}\right) p\left(\left\{y_{k}^{i}\right\}_{i \in N} \mid x_{k}\right) d\left\{y_{k}^{i}\right\}_{i \in N \backslash \mathcal{I}_{k}} \\
= & \int \prod_{i=1}^{N} p\left(\gamma_{k}^{i} \mid x_{k}, y_{k}^{i}\right) p\left(y_{k}^{i} \mid x_{k}\right) d\left\{y_{k}^{i}\right\}_{i \in N \backslash \mathcal{I}_{k}} \\
= & \prod_{i \in \mathcal{I}_{k}} p\left(\gamma_{k}^{i}=1 \mid x_{k}, y_{k}^{i}\right) p\left(y_{k}^{i} \mid x_{k}\right) \prod_{i \in N \backslash \mathcal{I}_{k}} p\left(\gamma_{k}^{i}=0 \mid x_{k}\right) \\
= & \prod_{i=1}^{N}\left[p\left(y_{k}^{i} \mid x_{k}\right)\right]^{\gamma_{k}^{i}}\left[p\left(\gamma_{k}^{i}=0 \mid x_{k}\right)\right]^{1-\gamma_{k}^{i}}
\end{aligned}
$$

where $N \backslash \mathcal{I}_{k}$ denotes the complement of $\mathcal{I}_{k}$ in the set $\{1,2, \ldots, N\}$. Note that the first equality means to successively integrate $p\left(\Gamma_{k},\left\{y_{k}^{i}\right\}_{i \in \mathcal{I}_{k}},\left\{y_{k}^{i}\right\}_{i \in N \backslash \mathcal{I}_{k}} \mid x_{k}\right)$ with respect to each element $y_{k}^{i}$ in the set $\left\{y_{k}^{i}\right\}_{i \in N \backslash \mathcal{I}_{k}}$. It is clear that the term $p\left(y_{k}^{i} \mid x_{k}\right)$ in (10) is directly determined by the measurement model (2), but the calculation of the term $p\left(\gamma_{k}^{i}=0 \mid x_{k}\right)$ is, unfortunately, non-trivial. In what follows, we are devoted to the derivation of an explicit formula for calculating the term $p\left(\gamma_{k}^{i}=0 \mid x_{k}\right)$ induced by the dynamic ETCM.

According to (5), $\gamma_{k}^{i}=0$ implies that $g\left(r_{k}^{i}, \varrho_{k}^{i}, \theta_{k}^{i}\right) \leq 0$, i.e.,

$$
\left\|r_{k}^{i}\right\|_{S^{i}}^{2} \leq \sigma^{i}+\frac{1}{\theta^{i}} \varrho_{k}^{i}
$$

Due to the fact that the auxiliary variable $\varrho_{k}^{i}$ for the $i$ th sensor node is unavailable to the central node, it is impossible to use (11) directly. Therefore, the following lemma is introduced.

Lemma 1: Under the dynamic ETCM described by (3)-(5), the following inequality holds for all $k>0$ :

$$
\left\|r_{k}^{i}\right\|_{S^{i}}^{2} \leq \Xi_{k}^{i}
$$

where

$$
\Xi_{k}^{i}=\frac{\left(\chi^{i}\right)^{k}}{\theta^{i}} \varrho_{0}^{i}+\frac{\left[1-\left(\chi^{i}\right)^{k}\right] \sigma^{i}}{\left(1-\chi^{i}\right) \theta^{i}}+\sigma^{i} .
$$

Proof: Recalling (4) and noticing that $\left\|r_{k-1}^{i}\right\|_{S^{i}}^{2} \geq 0$, one has

$$
\varrho_{k}^{i} \leq \chi^{i} \varrho_{k-1}^{i}+\sigma^{i} .
$$


This article has been accepted for publication in a future issue of this journal, but has not been fully edited. Content may change prior to final publication. Citation information: DOI10.1109/TSP.2020.3042947, IEEE Transactions on Signal Processing

FINAL VERSION

Substituting (13) into (11) yields

$$
\begin{aligned}
\left\|r_{k}^{i}\right\|_{S^{i}}^{2} & \leq \frac{\chi^{i}}{\theta^{i}} \varrho_{k-1}^{i}+\frac{\sigma^{i}}{\theta^{i}}+\sigma^{i} \\
& \leq \cdots \leq \frac{\left(\chi^{i}\right)^{k}}{\theta^{i}} \varrho_{0}^{i}+\left[\sum_{m=0}^{k-1}\left(\chi^{i}\right)^{m}\right] \frac{\sigma^{i}}{\theta^{i}}+\sigma^{i} \\
& =\frac{\left(\chi^{i}\right)^{k}}{\theta^{i}} \varrho_{0}^{i}+\frac{\left[1-\left(\chi^{i}\right)^{k}\right] \sigma^{i}}{\left(1-\chi^{i}\right) \theta^{i}}+\sigma^{i},
\end{aligned}
$$

which completes the proof.

Based on Lemma 1, it is inferred from $\gamma_{k}^{i}=0$ that the untransmitted measurement satisfies (12), i.e.,

$$
p\left(\gamma_{k}^{i}=0 \mid x_{k}\right)=p\left(\left\|r_{k}^{i}\right\|_{S^{i}}^{2} \leq \Xi_{k}^{i} \mid x_{k}\right) .
$$

As a consequence, the full likelihood function for the central node is rewritten as

$$
\begin{aligned}
& p\left(\Gamma_{k},\left\{y_{k}^{i}\right\}_{i \in \mathcal{I}_{k}} \mid x_{k}\right) \\
= & \prod_{i=1}^{N}\left[p\left(y_{k}^{i} \mid x_{k}\right)\right]^{\gamma_{k}^{i}}\left[p\left(\gamma_{k}^{i}=0 \mid x_{k}\right)\right]^{1-\gamma_{k}^{i}} \\
= & \prod_{i=1}^{N}\left[p\left(y_{k}^{i} \mid x_{k}\right)\right]^{\gamma_{k}^{i}}\left[p\left(\left\|r_{k}^{i}\right\|_{S^{i}}^{2} \leq \Xi_{k}^{i} \mid x_{k}\right)\right]^{1-\gamma_{k}^{i}}
\end{aligned}
$$

Now, the implementation of the proposed centralized auxiliary particle filtering under the dynamic event-triggered mechanism is summarized in Algorithm 1.

Remark 3: It is worth mentioning that the analytical expression of the probability $p\left(\left\|r_{k}^{i}\right\|_{S^{i}}^{2} \leq \Xi_{k}^{i} \mid x_{k}\right)$ can only be obtained in some special cases. For example, let us consider the case where the measurement output of the $i$ th sensor node is a scalar and the measurement noise $\nu_{k}^{i}$ follows a Gaussian distribution with mean $\mu_{k}^{i}$ and variance $\left(\rho_{k}^{i}\right)^{2}$. Then, we have

$$
\begin{aligned}
& p\left(\left\|r_{k}^{i}\right\|_{S^{i}}^{2} \leq \Xi_{k}^{i} \mid x_{k}\right) \\
= & p\left(-\sqrt{\Xi_{k}^{i} / S^{i}} \leq r_{k}^{i} \leq \sqrt{\Xi_{k}^{i} / S^{i}} \mid x_{k}\right) \\
= & p\left(\bar{y}_{k}^{i}-\sqrt{\Xi_{k}^{i} / S^{i}} \leq y_{k}^{i} \leq \bar{y}_{k}^{i}+\sqrt{\Xi_{k}^{i} / S^{i}} \mid x_{k}\right) \\
= & p\left(\underline{\nu}_{k}^{i} \leq \nu_{k}^{i} \leq \bar{\nu}_{k}^{i} \mid x_{k}\right) \\
= & \Phi\left(\frac{\bar{\nu}_{k}^{i}-\mu_{k}^{i}}{\rho_{k}^{i}}\right)-\Phi\left(\frac{\underline{\nu}_{k}^{i}-\mu_{k}^{i}}{\rho_{k}^{i}}\right)
\end{aligned}
$$

where

$$
\begin{aligned}
& \underline{\nu}_{k}^{i}=\bar{y}_{k}^{i}-h^{i}\left(x_{k}\right)-\sqrt{\Xi_{k}^{i} / S^{i}}, \\
& \bar{\nu}_{k}^{i}=\bar{y}_{k}^{i}-h^{i}\left(x_{k}\right)+\sqrt{\Xi_{k}^{i} / S^{i}}
\end{aligned}
$$

and $\Phi(\cdot)$ denotes the cumulative distribution function of the standard normal distribution. However, the above-mentioned method is not applicable when it comes to the case of vector measurement. Similar to [35], a Monte Carlo-based method is used to calculate the probability $p\left(\left\|r_{k}^{i}\right\|_{S^{i}}^{2} \leq \Xi_{k}^{i} \mid x_{k}\right)$ approximately. To be more specific, for each particle $x_{k}^{m}$, we draw $L$ samples denoted as $\left\{y_{k}^{i, m, l}\right\}_{l=1}^{L}$. Then, from the density $p\left(y_{k}^{i} \mid x_{k}^{m}\right)$, the probability $p\left(\left\|r_{k}^{i}\right\|_{S^{i}}^{2} \leq \Xi_{k}^{i} \mid x_{k}^{m}\right)$ can be approximated as

$$
p\left(\left\|r_{k}^{i}\right\|_{S^{i}}^{2} \leq \Xi_{k}^{i} \mid x_{k}^{m}\right)=\frac{1}{L} \sum_{l=1}^{L} 1_{\left\{\left\|r_{k}^{i, m, l}\right\|_{S^{i}}^{2} \leq \Xi_{k}^{i}\right\}}
$$

$\overline{\text { Algorithm } 1 \text { Centralized auxiliary particle filtering under the }}$ dynamic event-triggered mechanism

\section{Step 1. Particle initialization}

Draw particles $\left\{x_{0}^{m}\right\}_{m=1}^{M}$ from the prior density $p\left(x_{0}\right)$ and the associated importance weights $\left\{W_{0}^{m}\right\}_{m=1}^{M}$ are all set as $\frac{1}{M}$. In addition, the maximum recursive time instant is set as $K$.

Step 2. Measurement collection

Collect the measurements and the indicator variables at time instant $k$, i.e., $\left\{y_{k}^{i}\right\}_{i \in \mathcal{I}_{k}}$ and $\Gamma_{k}$, under the dynamic ETCM described by (3)-(5).

Step 3. Particle selection and update

Obtain the indices $i n d_{m}$ according to the probabilities proportional to $W_{k-1}^{m} p\left(\Gamma_{k},\left\{y_{k}^{i}\right\}_{i \in \mathcal{I}_{k}} \mid \lambda_{k}^{m}\right)$, where $\lambda_{k}^{m} \sim p\left(x_{k} \mid x_{k-1}^{m}\right)$ and $p\left(\Gamma_{k},\left\{y_{k}^{i}\right\}_{i \in \mathcal{I}_{k}} \mid \lambda_{k}^{m}\right)$ is calculated by (16); Draw new particle $x_{k}^{m}$ from the transition PDF $p\left(x_{k} \mid x_{k-1}^{i n d_{m}}\right)$ for $m=1, \ldots, M$.

Step 4. Weight assignment

Assign the unnormalized weights $\left\{\tilde{W}_{k}^{m}\right\}_{m=1}^{M}$ according to

$$
\tilde{W}_{k}^{m}=\frac{p\left(\Gamma_{k},\left\{y_{k}^{i}\right\}_{i \in \mathcal{I}_{k}} \mid x_{k}^{m}\right)}{p\left(\Gamma_{k},\left\{y_{k}^{i}\right\}_{i \in \mathcal{I}_{k}} \mid \lambda_{k}^{i n d_{m}}\right)} .
$$

Step 5. Normalization

Normalize the importance weights as $W_{k}^{m}=$ $\tilde{W}_{k}^{m} /\left(\sum_{n=1}^{M} \tilde{W}_{k}^{n}\right)$.

Step 6. State estimate update

Calculate the state estimate $\hat{x}_{k}$ and estimation error covariance $P_{k}$ as

$$
\begin{aligned}
& \hat{x}_{k}=\sum_{m=1}^{M} W_{k}^{m} x_{k}^{m}, \\
& P_{k}=\sum_{m=1}^{M} W_{k}^{m}\left(x_{k}^{m}-\hat{x}_{k}\right)\left(x_{k}^{m}-\hat{x}_{k}\right)^{T} .
\end{aligned}
$$

Step 7. If $k<K$, then set $k=k+1$ and go to Step 2; otherwise go to Step 8.

Step 8. Stop.

where

$$
r_{k}^{i, m, l}=y_{k}^{i, m, l}-\bar{y}_{k}^{i}
$$

and

$$
1_{\left\{\left\|r_{k}^{i, m, l}\right\|_{S^{i}}^{2} \leq \Xi_{k}^{i}\right\}}
$$

is the indicator function defined as

$$
1_{\left\{\left\|r_{k}^{i, m, l}\right\|_{S^{i}}^{2} \leq \Xi_{k}^{i}\right\}}= \begin{cases}1, & \text { if }\left\|r_{k}^{i, m, l}\right\|_{S^{i}}^{2} \leq \Xi_{k}^{i} \\ 0, & \text { otherwise. }\end{cases}
$$

\section{B. Distributed auxiliary particle filtering under the dynamic event-triggered mechanism}

Due to the limited network bandwidth and energy supply, the centralized particle filtering algorithm proposed in Section III-A, which requires the all-to-one communication, is clearly impractical in large-scale and wide-area sensor network. For example, when the central node fails to work well 


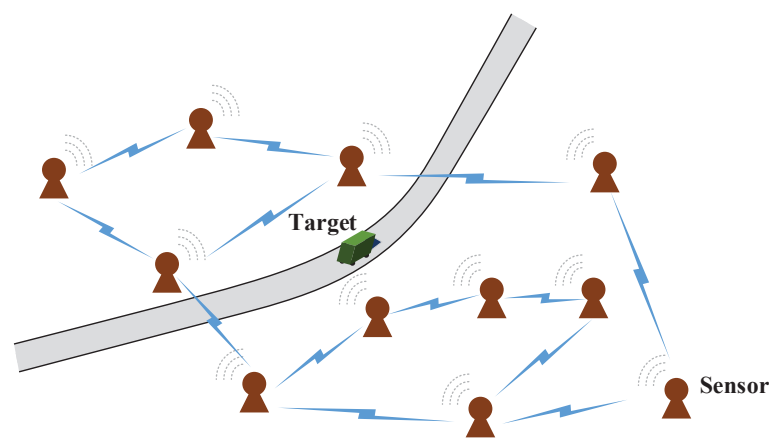

Fig. 1: The system model of the distributed inference problem.

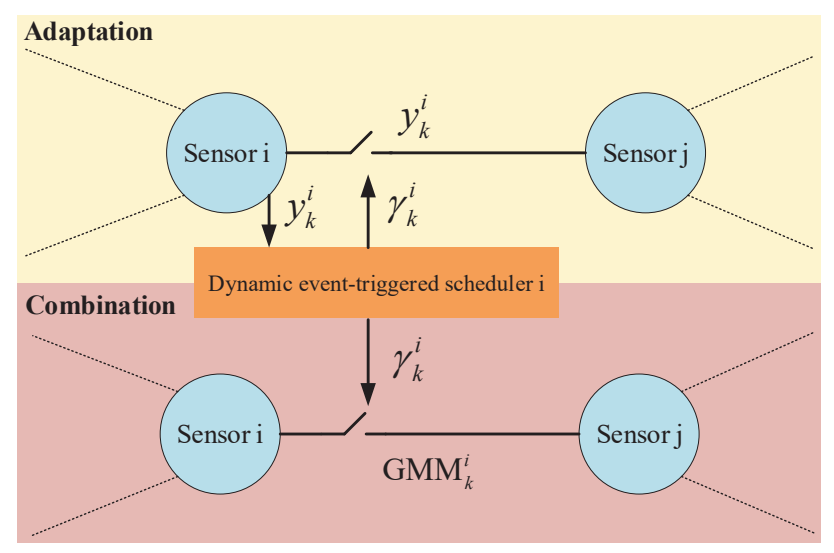

Fig. 2: Internode communication in the diffusion strategy under the dynamic event-triggered mechanism.

(e.g., attacked by the adversaries) and becomes unavailable, the whole system may be paralyzed. Hence, it is more desirable to develop a distributed filtering algorithm to eliminate the dependence on the central node. The system model is depicted in Fig. 1.

In this section, a distributed particle filtering algorithm under the dynamic ETCM is proposed by utilizing the adaptthen-combine diffusion strategy [9], [11], where each node only communicates with its neighboring nodes and no central node is required. The proposed algorithm is sketched in Fig. 2, where the notation $\mathrm{GMM}_{k}^{i}$ denotes the Gaussian mixture model representation for the posterior PDF of sensor node $i$ at time instant $k$, see (27) for the definition. The detailed algorithm is explained as follows.

1) Adaptation Stage: For sensor node $i$, the available measurement information at time instant $k$ is denoted by

$$
\begin{aligned}
Z_{k}^{i} & =\left\{y_{k}^{j}: j \in \mathcal{I}_{k}^{i}\right\}, \\
\Gamma_{k}^{i} & =\left\{\gamma_{k}^{i j}: j \in \mathcal{N}_{i}\right\}
\end{aligned}
$$

where $\mathcal{N}_{i}$ stands for the set of sensor nodes connected to node $i$ and $\mathcal{I}_{k}^{i} \subseteq \mathcal{N}_{i}$ represents the set of neighboring nodes that transmit their current measurements to node $i$ at time instant $k$. Note that $\gamma_{k}^{i j}=\gamma_{k}^{j}$ if $j \in \mathcal{N}_{i} \backslash i$, and $\gamma_{k}^{i j}=1$ if $j=i$. It is worth mentioning that $i \in \mathcal{I}_{k}^{i}$ and $i \in \mathcal{N}_{i}$. All available measurement information up to time instant $k$ is written by

$$
\begin{aligned}
Z_{1: k}^{i} & =\left\{Z_{1}^{i}, Z_{2}^{i}, \cdots, Z_{k}^{i}\right\}, \\
\Gamma_{1: k}^{i} & =\left\{\Gamma_{1}^{i}, \Gamma_{2}^{i}, \cdots, \Gamma_{k}^{i}\right\} .
\end{aligned}
$$

In this stage, the statistical knowledge of sensor node $i$ is enriched by incorporating its neighboring nodes' measurements. Specifically, sensor node $i$ collects all available measurement information and runs, independently, a local particle filter to obtain a particle-based approximation of the posterior PDF $p\left(x_{k} \mid \Gamma_{1: k}^{i}, Z_{1: k}^{i}\right)$.

Let the particles $\left\{x_{k-1}^{i, m}\right\}_{m=1}^{M}$ and associated importance weights $\left\{W_{k-1}^{i, m}\right\}_{m=1}^{M}$ for node $i$ at time instant $k-1$ be all known. At time instant $k$, the first step is to obtain the indices $i n d_{i, m}$ according to the probabilities proportional to $W_{k-1}^{i, m} p\left(\Gamma_{k}^{i}, Z_{k}^{i} \mid \lambda_{k}^{i, m}\right)$, where $\lambda_{k}^{i, m} \sim p\left(x_{k} \mid x_{k-1}^{i, m}\right)$ and $p\left(\Gamma_{k}^{i}, Z_{k}^{i} \mid \lambda_{k}^{i, m}\right)$ is calculated by

$$
\begin{aligned}
& p\left(\Gamma_{k}^{i}, Z_{k}^{i} \mid \lambda_{k}^{i, m}\right) \\
= & \prod_{j \in \mathcal{N}_{i}}\left[p\left(y_{k}^{j} \mid \lambda_{k}^{i, m}\right)\right]^{i j}\left[p\left(\gamma_{k}^{j}=0 \mid \lambda_{k}^{i, m}\right)\right]^{1-\gamma_{k}^{i j}} \\
= & \prod_{j \in \mathcal{N}_{i}}\left[p\left(y_{k}^{j} \mid \lambda_{k}^{i, m}\right)\right]^{i j}\left[p\left(\left\|r_{k}^{j}\right\|_{S^{j}}^{2} \leq \Xi_{k}^{j} \mid \lambda_{k}^{i, m}\right)\right]^{1-\gamma_{k}^{i j}} .
\end{aligned}
$$

Based on the indices $i n d_{i, m}$, the next step is to sample new particles $\left\{x_{k}^{i, m}\right\}_{m=1}^{M}$ from the prior density, i.e.,

$$
x_{k}^{i, m} \sim p\left(x_{k} \mid x_{k-1}^{i n d_{i, m}}\right)
$$

and update the weights $\left\{W_{k}^{i, m}\right\}_{m=1}^{M}$ by

$$
W_{k}^{i, m}=\frac{p\left(\Gamma_{k}^{i}, Z_{k}^{i} \mid x_{k}^{i, m}\right)}{p\left(\Gamma_{k}^{i}, Z_{k}^{i} \mid \lambda_{k}^{i n d_{i, m}}\right)}
$$

where $p\left(\Gamma_{k}^{i}, Z_{k}^{i} \mid x_{k}^{i, m}\right)$ and $p\left(\Gamma_{k}^{i}, Z_{k}^{i} \mid \lambda_{k}^{i n d_{i, m}}\right)$ are similarly calculated according to (21). In view of (22) and (23), the particle representation of the local posterior PDF $p\left(x_{k} \mid \Gamma_{1: k}^{i}, Z_{1: k}^{i}\right)$ is obtained.

2) Combination Stage: In this stage, each sensor node shares the local posterior PDF obtained in the adaptation stage with its neighboring nodes and merges the information received from the neighboring nodes to collaboratively improve the estimation accuracy. As illustrated in [17], the fused posterior PDF at sensor node $i$ (denoted as $\hat{p}\left(x_{k} \mid \Gamma_{1: k}^{i}, Z_{1: k}^{i}\right)$ ) can be written as

$$
\hat{p}\left(x_{k} \mid \Gamma_{1: k}^{i}, Z_{1: k}^{i}\right) \propto \prod_{j \in \mathcal{N}_{i}}\left[p\left(x_{k} \mid \Gamma_{1: k}^{j}, Z_{1: k}^{j}\right)\right]^{\beta_{i j}},
$$

which is the one that minimizes the weighted average Kullback-Leibler divergence

$$
\sum_{j \in \mathcal{N}_{i}} \beta_{i j} D_{K L}\left\{p^{\prime}\left(x_{k} \mid \Gamma_{1: k}^{i}, Z_{1: k}^{i}\right) \| p\left(x_{k} \mid \Gamma_{1: k}^{j}, Z_{1: k}^{j}\right)\right\}
$$

over all the candidates $p^{\prime}\left(x_{k} \mid \Gamma_{1: k}^{i}, Z_{1: k}^{i}\right)$, where $D_{K L}\{\cdot \| \cdot\}$ stands for the Kullback-Leibler divergence between two distributions, and the weight $\beta_{i j}$ represents the belief degree of node $i$ in the information of node $j$ and satisfies $\sum_{j \in \mathcal{N}_{i}} \beta_{i j}=1$. 
This article has been accepted for publication in a future issue of this journal, but has not been fully edited. Content may change prior to final publication. Citation information: DOI10.1109/TSP.2020.3042947, IEEE Transactions on Signal Processing

In practice, by taking both the information of the communication topology and the effect of the dynamic ETCM into consideration, the weight $\beta_{i j}$ can be selected as

$$
\beta_{i j}= \begin{cases}\frac{1}{\left|\mathcal{I}_{k}^{i}\right|}, & \text { if } j \in \mathcal{N}_{i} \text { and } \gamma_{k}^{i j}=1 \text { (i.e., } j \in \mathcal{I}_{k}^{i} \text { ) } \\ 0, & \text { otherwise }\end{cases}
$$

where $\left|\mathcal{I}_{k}^{i}\right|$ denotes the cardinality of $\mathcal{I}_{k}^{i}$.

Remark 4: Note that, in the combination stage, the sensor node $i$ needs to share its local posterior PDF with its neighboring nodes, which requires the transmission of particles and their respective importance weights. In this case, $(n+1) M$ real numbers (i.e., $n M$ numbers for particles and $M$ numbers for weights) are required to be transmitted for each node at each time instant (if triggered). With the increase of the particle numbers, the communication cost will become considerably high. Hence, it is often preferable to present and broadcast the local posterior PDF in a parametric fashion. Notice that additional estimation errors might be induced by using the single Gaussian distribution to approximate the non-Gaussian posterior PDF. Therefore, the more accurate Gaussian mixture representation has been widely used in the literature [1], [10]. With the Gaussian mixture representation of the local posterior, each sensor node only needs to transmit $G\left(1+n+n^{2}\right)$ numbers, where $G$ is the average number of Gaussian components in the Gaussian mixture models. Then, the maximum communication load under the diffusion strategy is $\mathcal{O}\left(N(N-1)\left(G\left(1+n+n^{2}\right)+m\right)\right)$, where $m$ is the measurement dimension. In fact, the communication load can be further reduced by considering the symmetry of the covariance matrices.

In this paper, the local posterior PDF for sensor node $i$ is represented by a $G_{i}$-component Gaussian mixture model

$$
p\left(x_{k} \mid Z_{1: k}^{i}, \Gamma_{1: k}^{i}\right)=\sum_{g=1}^{G_{i}} a_{g} \mathbb{N}\left(x_{k} ; \mu_{g}, \Sigma_{g}\right) \triangleq \mathrm{GMM}_{k}^{i}
$$

where $\mathbb{N}\left(x_{k} ; \mu_{g}, \Sigma_{g}\right)$ denotes the $g$ th component with $a_{g}$ being its weight. To obtain a Gaussian mixture representation for the posterior PDF required in the combination stage, the weighted expectation-maximization algorithm detailed in [1] is employed. Moreover, it should be noticed from the combination rule (24) that the fusion of Gaussian mixture models with non-integer exponents is involved. In this paper, the weighted mixture importance sampling method [29] is adopted to realize such a fusion.

The proposed distributed filtering scheme is subsequently outlined in Algorithm 2, where only the operations conducted by sensor node $i$ are displayed.

Remark 5: Since the filtering process has similar computational burden among different algorithms, we only focus on the most computation-intensive operations, i.e., the Gaussian mixture representation learning for the local posterior in the adaptation stage and the fusion of local posteriors in the combination stage. Note that even though the dynamic ETCM is introduced to schedule the data transmission among sensor nodes, we consider the worst case in the analysis, i.e., each sensor is able to connect with $N$ neighbors (including itself). $\overline{\text { Algorithm } 2 \text { Diffusion-based distributed auxiliary particle }}$ filtering under the dynamic ETCM (executed on sensor node i)

Step 1. Particle initialization

Draw particles $\left\{x_{0}^{i, m}\right\}_{m=1}^{M}$ from the prior density $p\left(x_{0}\right)$ and the associated importance weights $\left\{W_{0}^{i, m}\right\}_{m=1}^{M}$ are all set as $\frac{1}{M}$. In addition, the maximum recursive time instant is set as $K$.

Step 2. Measurement collection

Sensor node $i$ assimilates the available measurements from its neighboring nodes at time instant $k$, i.e., $\left\{y_{k}^{i}\right\}_{i \in \mathcal{I}_{k}^{i}}$ and $\Gamma_{k}^{i}$, and broadcasts its current measurements to its neighbors under the dynamic ETCM.

Step 3. Particle selection and update

Select particles $x_{k-1}^{i n d_{i, m}}$ based on (21) and propagate particles $x_{k-1}^{i n d_{i, m}}$ to $x_{k}^{i, m}$ for $m=1, \ldots, M$ according to (22).

Step 4. Weight assignment

Assign the unnormalized weights $\left\{\tilde{W}_{k}^{i, m}\right\}_{m=1}^{M}$ according to (23).

Step 5. Normalization

Normalize the importance weights as $W_{k}^{i, m}=$ $\tilde{W}_{k}^{i, m} /\left(\sum_{n=1}^{M} \tilde{W}_{k}^{i, n}\right)$.

Step 6. Gaussian mixture model representation

Construct the Gaussian mixture model $\mathrm{GMM}_{k}^{i}$ based on the weighted particles $\left\{x_{k}^{i, m}, W_{k}^{i, m}\right\}_{m=1}^{M}$ by using the weighted expectation-maximization algorithm.

Step 7. Local posteriors sharing

Sensor node $i$ shares its local posterior represented by $\mathrm{GMM}_{k}^{i}$ with its neighbors according to the indicator variable $\gamma_{k}^{i}$, and receives the local posteriors from its neighbors.

Step 8. Combination

Utilize the weighted mixture importance sampling method to merge the Gaussian mixture models according to (25), and extract the state estimate $\hat{x}_{k}^{i}$ as

$$
\hat{x}_{k}^{i}=\sum_{m=1}^{M} W_{k}^{i, m} x_{k}^{i, m}
$$

where $\left\{x_{k}^{i, m}, W_{k}^{i, m}\right\}_{m=1}^{M}$ are newly sampled from the merged Gaussian mixture model.

Step 9. If $k<K$, then set $k=k+1$ and go to Step 2; otherwise go to Step 10.

Step 10. Stop. 
This article has been accepted for publication in a future issue of this journal, but has not been fully edited. Content may change prior to final publication. Citation information: DOI10.1109/TSP.2020.3042947, IEEE Transactions on Signal Processing

FINAL VERSION

Similar to [29], it can be concluded that the main computational complexity of the proposed distributed filtering algorithm executed on each sensor node is $\mathcal{O}\left(K(N+I) M G n^{2}\right)$, where $I$ denotes the number of learning iterations in the weighted expectation-maximization algorithm, and $G$ is the average number of Gaussian components in the Gaussian mixture models.

Remark 6: So far, we have dealt with the distributed auxiliary particle filtering problem for a class of general nonlinear/non-Gaussian systems where the data transmissions are triggered in a dynamical fashion. More specifically, we have developed dynamic event-triggered particle filters where the dynamic ETCM is subtly embedded for mitigating dataintensive transmissions. We have also examined the effect from the dynamic ETCM on the performance of the proposed filtering schemes. Furthermore, we have made full use of the information from the non-triggered measurements to assist in selecting the candidate particles and updating weights.

Remark 7: In this paper, the proposed centralized and distributed auxiliary particle filtering algorithms under the dynamic ETCM have been, respectively, summarized in Algorithms 1 and 2. It is clear from Algorithm 1 that each sensor (if triggered) is required to transmit its current measurement to a fusion center, and the fusion center runs a modified auxiliary particle filter to obtain the state estimate. Such a scheme may consume a large amount of communication resource and may fail to function especially when the fusion center suffers from critical failure. For the distributed counterpart described in Algorithm 2, each sensor only needs to communicate with its neighboring nodes on the basis of the dynamic ETCM, and the fusion center disappears. Specifically, each sensor first receives the triggered measurements from its neighbors and runs a modified auxiliary particle filter to form the local posterior, and then fuses the available local posteriors to extract the state estimate.

Remark 8: For decades, the particle filtering algorithms have been known to be capable of handling nonlinear/nonGaussian systems and a great deal of research attention has been devoted to the applications on these algorithms to a variety of engineering practice. Comparing to existing results, the main results established in this paper stand out for the following reasons: 1) a new distributed auxiliary particle filter algorithm is developed based on the diffusion strategy for a class of general discrete-time nonlinear/non-Gaussian systems; 2) a dynamic ETCM is, for the first time, introduced in the particle filter design with hope to save energy consumption; and 3) non-triggered measurements are properly taken into account in order to further improve the performance of the developed particle filter algorithm.

\section{NUMERICAL STUdY}

In this section, the performance of the proposed centralized and distributed auxiliary particle filtering algorithms under the dynamic ETCM is demonstrated via the application in the moving target tracking problem using received-signal-strength measurements.

\section{A. Target tracking}

Assume that the target moves in a two-dimensional (2D) plane and its state vector is represented by $x_{k}=$ $\left[x_{k}^{t}, \dot{x}_{k}^{t}, y_{k}^{t}, \dot{y}_{k}^{t}\right]^{T}$, where $\left(x_{k}^{t}, y_{k}^{t}\right)$ and $\left(\dot{x}_{k}^{t}, \dot{y}_{k}^{t}\right)$ denote the target's 2-D position and 2-D velocity, respectively. The dynamics of the moving target is described by the well-known white noise acceleration model [18], i.e.,

$$
x_{k+1}=\left[\begin{array}{cccc}
1 & T & 0 & 0 \\
0 & 1 & 0 & 0 \\
0 & 0 & 1 & T \\
0 & 0 & 0 & 1
\end{array}\right] x_{k}+\omega_{k}
$$

where $T$ represents the sampling period and $\omega_{k}$ is the zeromean Gaussian white noise sequences with covariance matrix

$$
Q_{k}=\Upsilon\left[\begin{array}{cccc}
\frac{T^{3}}{3} & \frac{T^{2}}{2} & 0 & 0 \\
\frac{T^{2}}{2} & T & 0 & 0 \\
0 & 0 & \frac{T^{3}}{3} & \frac{T^{2}}{2} \\
0 & 0 & \frac{T^{2}}{2} & T
\end{array}\right]
$$

where $\Upsilon$ stands for the acceleration variance.

In the considered scenario, the moving target emits a radio or acoustic signal and we employ $N$ passive received-signalstrength sensors to measure the signal energy emitted by the target. The measurement characteristic of the $i$ th receivedsignal-strength sensor node located at $\left(x_{k}^{s, i}, y_{k}^{s, i}\right)$ is modeled by [18], [46]

$$
\begin{aligned}
y_{k}^{i}= & P_{0}-10 n_{r} \log _{10}\left(\frac{\left\|\left[x_{k}^{t}, y_{k}^{t}\right]^{T}-\left[x_{k}^{s, i}, y_{k}^{s, i}\right]^{T}\right\|}{d_{0}}\right) \\
& +\nu_{k}^{i}, \quad i=1,2, \ldots, N,
\end{aligned}
$$

where $\|\cdot\|$ stands for the Euclidean norm, $P_{0}$ denotes the received signal energy at the reference distance $d_{0}, n_{r}$ represents the path loss exponent, and $\nu_{k}^{i}$ is the measurement noise. For each sensor node $i$, we assume that $\nu_{k}^{i}$ is modeled by a mixture of two Gaussian distributions, i.e.,

$$
p\left(\nu_{k}^{i}\right)=\left(1-\pi^{i}\right) \mathbb{N}\left(\nu_{k}^{i} ; \mu_{i, 1}, \epsilon_{i, 1}^{2}\right)+\pi^{i} \mathbb{N}\left(\nu_{k}^{i} ; \mu_{i, 2}, \epsilon_{i, 2}^{2}\right)
$$

where $\pi^{i}$ is the glint probability.

As a popular measure of the filtering accuracy, the root mean-square error (RMSE) on position and velocity estimates are respectively calculated according to

$$
\begin{aligned}
\mathrm{RMSE}_{\mathrm{Pos}, k} & =\sqrt{\frac{1}{N_{M}} \sum_{j=1}^{N_{M}}\left[\left(x_{k}^{t, j}-\hat{x}_{k}^{t, j}\right)^{2}+\left(y_{k}^{t, j}-\hat{y}_{k}^{t, j}\right)^{2}\right]}, \\
\mathrm{RMSE}_{\mathrm{Vel}, k} & =\sqrt{\frac{1}{N_{M}} \sum_{j=1}^{N_{M}}\left[\left(\dot{x}_{k}^{t, j}-\hat{\dot{x}}_{k}^{t, j}\right)^{2}+\left(\dot{y}_{k}^{t, j}-\hat{\dot{y}}_{k}^{t, j}\right)^{2}\right]}
\end{aligned}
$$

where $\left(x_{k}^{t, j}, y_{k}^{t, j}\right)$ and $\left(\dot{x}_{k}^{t, j}, \dot{y}_{k}^{t, j}\right)$ denote the realization of $\left(x_{k}^{t}, y_{k}^{t}\right)$ and $\left(\dot{x}_{k}^{t}, \dot{y}_{k}^{t}\right)$ in the $j$ th Monte Carlo run, and their respective estimates are represented by $\left(\hat{x}_{k}^{t, j}, \hat{y}_{k}^{t, j}\right)$ and $\left(\hat{\dot{x}}_{k}^{t, j}, \hat{\dot{y}}_{k}^{t, j}\right) . N_{M}$ is the total number of the Monte Carlo runs. 
This article has been accepted for publication in a future issue of this journal, but has not been fully edited. Content may change prior to final publication. Citation information: DOI10.1109/TSP.2020.3042947, IEEE Transactions on Signal Processing

FINAL VERSION

\section{B. Simulation setups}

In each independent Monte Carlo run, the true state is initialized as $x_{0}=[25 \mathrm{~m}, 0.3 \mathrm{~m} / \mathrm{s}, 20 \mathrm{~m}, 0.4 \mathrm{~m} / \mathrm{s}]^{\mathrm{T}}$, the sampling period and the acceleration variance are chosen as $T=1 \mathrm{~s}$ and $\Upsilon=0.04^{2} \mathrm{~m}^{2} / \mathrm{s}^{4}$, respectively. Following [18], the position components of the initial particles are sampled from a Gaussian distribution $\mathbb{N}\left([25,20]^{T}, \operatorname{diag}\left\{30^{2}, 30^{2}\right\}\right)$ and the velocity components are sampled according to a Gaussian distribution $\mathbb{N}\left([0.5, \arctan (4 / 3)]^{T}, \operatorname{diag}\left\{0.5^{2},(5 \pi / 180)^{2}\right\}\right)$, which characterizes the initial resultant velocity and the azimuth. Furthermore, we have $N=6$ sensors deployed in the surveillance areas to observe the target of interest. The sensors parameters and other parameters related to the dynamic ETCM are listed in TABLE I for clarity purposes.

TABLE I: Parameter settings

\begin{tabular}{lccc}
\hline \hline Parameters & Values & Parameters & Values \\
\hline$P_{0}$ & $1 \mathrm{dBm}$ & $d_{0}$ & $1 \mathrm{~m}$ \\
$n_{r}$ & 2 & $\pi^{i}$ & 0.1 \\
$\mu_{i, 1}$ & $0 \mathrm{dBm}$ & $\mu_{i, 2}$ & $0 \mathrm{dBm}$ \\
$\epsilon_{i, 1}$ & $1.2 \mathrm{dBm}$ & $\epsilon_{i, 2}$ & $10 \epsilon_{i, 1} \mathrm{dBm}$ \\
$\varrho_{0}^{i}$ & 100 & $\sigma^{i}$ & 2 \\
$\theta^{i}$ & 5 & $\chi^{i}$ & 0.9 \\
$S^{i}$ & 1 & $K$ & 100 \\
$\mathrm{M}$ & 500 & $N_{M}$ & 20 \\
\hline \hline
\end{tabular}

In the simulation, the following five algorithms are evaluated and compared: 1) CAPF-DETM: the proposed centralized auxiliary particle filter under the dynamic ETCM (see Algorithm 1); 2) CAPF-DETM-WO: a centralized auxiliary particle filter under the dynamic ETCM without utilizing the information contained in the non-triggered measurements; 3 ) CPF-DETM: the framework of the auxiliary particle filter in Algorithm 1 is replaced by that of the sampling importance resampling (SIR) particle filter; 4) DAPF-DETM: the proposed diffusion-based distributed auxiliary particle filter under the dynamic ETCM (see Algorithm 2); and 5) DAPF-DETM-OA: a distributed auxiliary particle filter under the dynamic ETCM using the adaptation-only scheme.

\section{Simulation results}

In Fig. 3, one realization of the true trajectory and its estimate given by the CAPF-DETM, as well as the sensor positions are depicted, which indicates that the proposed CAPF-DETM is capable of achieving a satisfactory tracking performance. Moreover, it can be seen from Fig. 4 that the transmission times are significantly reduced under the dynamic ETCM. The evolutions of the RMSEs with respect to the position and velocity estimates calculated by the CAPF-DETM, CAPF-DETM-WO and CPF-DETM are respectively plotted in Figs. 5-6. As expected, the proposed centralized algorithm has the best tracking performance when compared with the other two algorithms. The reason is that we have made full use of the information contained in the non-triggered measurements, which contributes to selecting the most promising particles.

In the following, we aim to examine the performance of our proposed DAPF-DETM. The communication topology of the

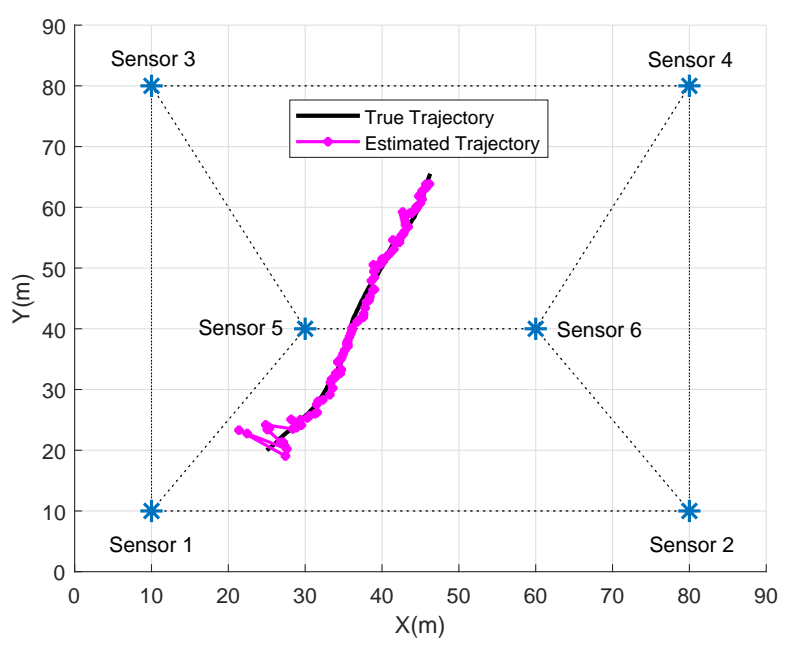

Fig. 3: The true target trajectory and its estimate obtained by the CAPF-DETM in one run.

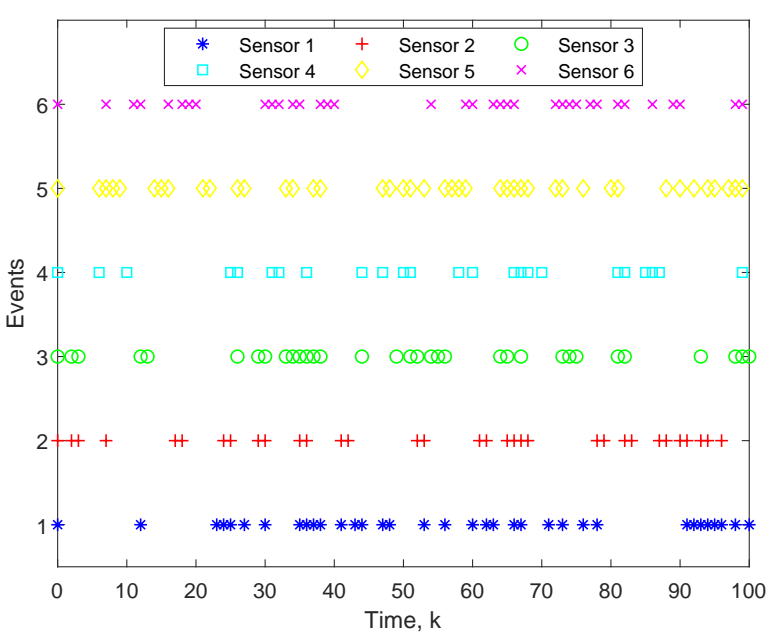

Fig. 4: The triggering instants.

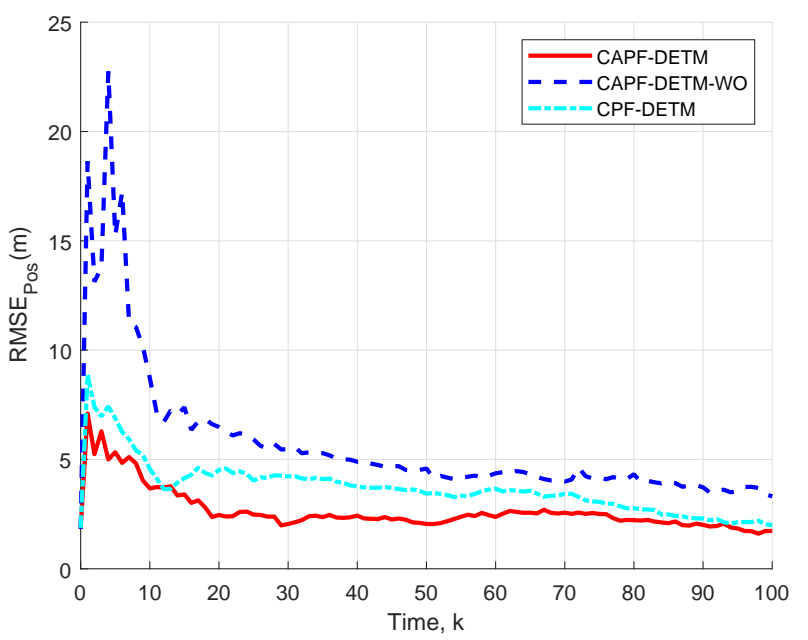

Fig. 5: Position RMSEs of CAPF-DETM, CAPF-DETM-WO and CPF-DETM. 
This article has been accepted for publication in a future issue of this journal, but has not been fully edited. Content may change prior to final publication. Citation information: DOI10.1109/TSP.2020.3042947, IEEE Transactions on Signal Processing

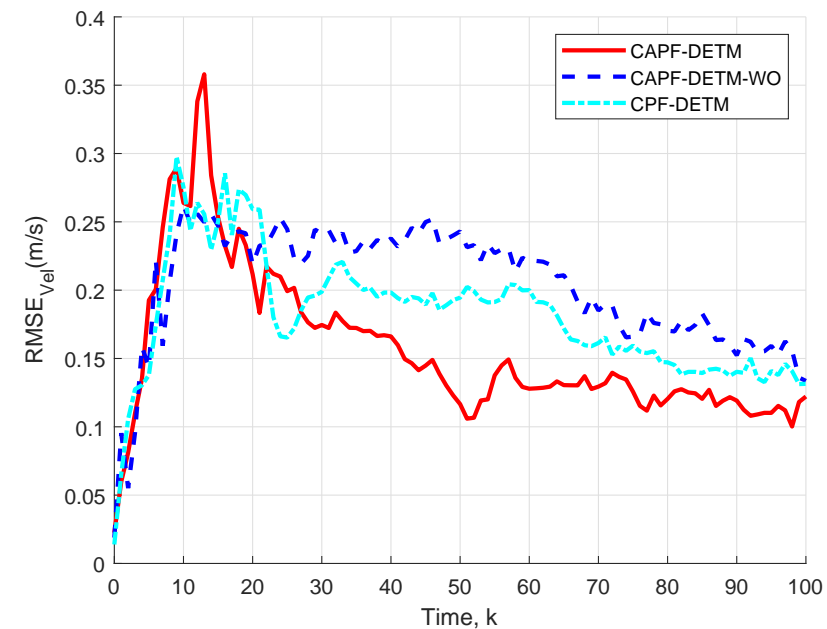

Fig. 6: Velocity RMSEs of CAPF-DETM, CAPF-DETM-WO and CPF-DETM.

sensor network modeled by an undirected graph is illustrated in Fig. 3. The trajectories of RMSEs on the position and velocity estimates obtained from the CAPF-DETM, DAPF-DETM and DAPF-DETM-OA are presented in Figs. 7-8. It should be noted that in some instants, the DAPF-DETM can even perfor$\mathrm{m}$ better than the CAPF-DETM probably due to the reason that when all sensors are not triggered, each sensor still has access to its own measurements in the distributed implementation. On the other hand, we can find that the DAPF-DETM-OA incurs larger performance degradation when compared with DAPFDETM owing to the absence of combination step. In fact, the adaptation-only scheme just updates the local estimates by utilizing the neighbors' measurements under the dynamic ETCM, which fails to diffuse the estimates throughout the whole network.

Further simulations are conducted to analyze the effect of the parameter $\theta^{i}$ (the superscript $i$ is omitted next for conciseness) in the dynamic ETCM on the transmission performance and tracking performance. As an indicator of transmission performance, the triggering rate is defined as the ratio of the actual triggering times to the total length of the simulation period. The average triggering rate $\mathcal{R}$ is the mean of the triggering rate over the $N_{M}$ Monte Carlo runs. In addition, the average RMSE on position (respectively, velocity), denoted as $\mathcal{E}_{\mathrm{Pos}}$ (respectively, $\mathcal{E}_{\mathrm{Vel}}$ ), is the mean of the $\mathrm{RMSE}_{\mathrm{Pos}, k}$ (respectively, $\mathrm{RMSE}_{\mathrm{Vel}, k}$ ) over the total simulation period. The corresponding results of three representative sensors are shown in TABLE II and Figs. 9-10.

From TABLE II, it appears that the average triggering rate decreases as the parameter $\theta$ decreases. Recalling that the case with $\theta=\infty$ is equivalent to the static event-triggered mechanism, we can naturally conclude that the dynamic ETCM can schedule the data transmission in a more efficient way. On the other hand, the results presented in TABLE II and Figs. 9-10 also imply that the choice of parameter $\theta$ is of great importance for the filtering accuracy. It should be pointed out that as the parameter $\theta$ decreases, the RMSEs will increase. Therefore,

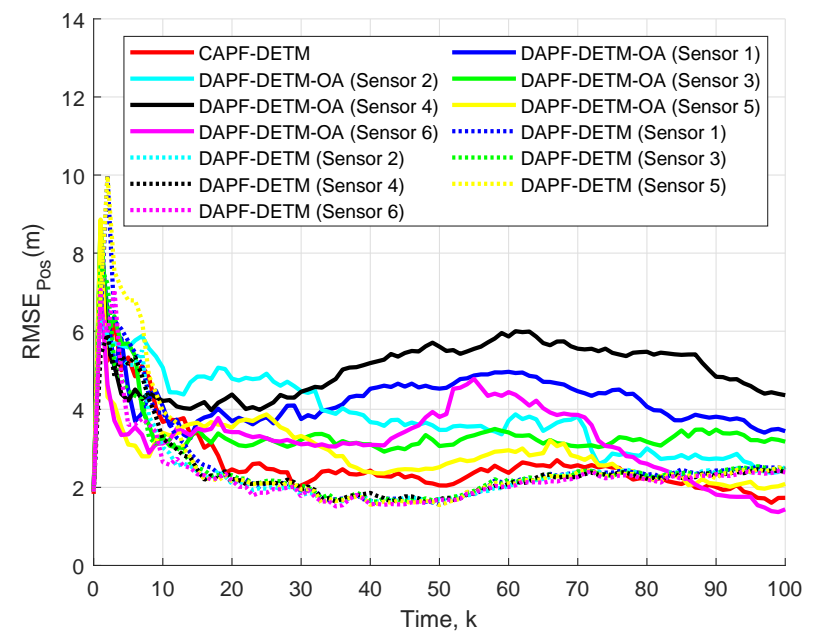

Fig. 7: Position RMSEs of CAPF-DETM, DAPF-DETM and DAPF-DETM-OA.

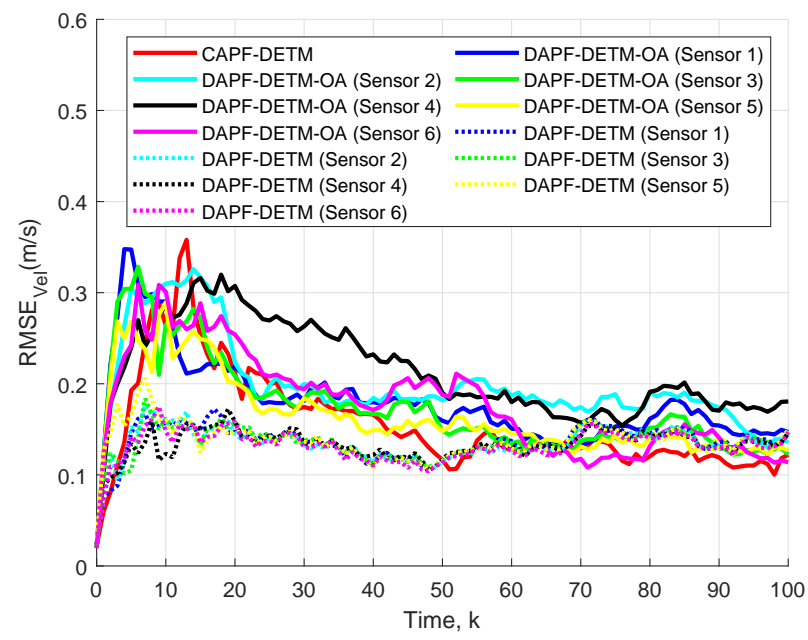

Fig. 8: Velocity RMSEs of CAPF-DETM, DAPF-DETM and DAPF-DETM-OA.

a trade-off between the communication cost and the filtering performance can be achieved via the parameter adjustment, which provides more flexibility to the practical applications.

\section{CONClusions}

In this paper, the diffusion-based distributed auxiliary particle filtering algorithm has been proposed to solve the nonlinear/non-Gaussian filtering problem under the dynamic ETCM. Compared with the traditional static event-triggered mechanism, the dynamic counterpart can automatically adjust the equivalent triggering threshold and has more potential to reduce the communication energy consumption. A full likelihood function has been firstly established in the centralized auxiliary particle filtering algorithm by using not only the transmitted measurements but also the information mined from the non-triggered measurements, which contributes to selecting the most promising candidate particles and updating the weights. In the distributed auxiliary particle filtering algorithm, 
This article has been accepted for publication in a future issue of this journal, but has not been fully edited. Content may change prior to final publication. Citation information: DOI10.1109/TSP.2020.3042947, IEEE Transactions on Signal Processing

TABLE II: The effect of the parameter on the average triggering rate and average RMSEs

\begin{tabular}{|c|c|c|c|c|c|c|c|c|c|}
\hline & \multicolumn{3}{|c|}{ Sensor 1} & \multicolumn{3}{|c|}{ Sensor 3} & \multicolumn{3}{|c|}{ Sensor 5} \\
\hline & $\mathcal{R}$ & $\mathcal{E}_{\text {Pos }}(\mathrm{m})$ & $\mathcal{E}_{\mathrm{Vel}}(\mathrm{m} / \mathrm{s})$ & $\mathcal{R}$ & $\mathcal{E}_{\text {Pos }}(\mathrm{m})$ & $\mathcal{E}_{\mathrm{Vel}}(\mathrm{m} / \mathrm{s})$ & $\mathcal{R}$ & $\mathcal{E}_{\text {Pos }}(\mathrm{m})$ & $\mathcal{E}_{\mathrm{Vel}}(\mathrm{m} / \mathrm{s})$ \\
\hline$\theta=5$ & $33.47 \%$ & 2.5308 & 0.1354 & $33.42 \%$ & 2.4306 & 0.1354 & $33.32 \%$ & 2.5658 & 0.1369 \\
\hline$\theta=15$ & $40.99 \%$ & 2.2445 & 0.1341 & $43.07 \%$ & 2.2430 & 0.1346 & $41.14 \%$ & 2.2493 & 0.1321 \\
\hline$\theta=\infty$ & $49.75 \%$ & 1.8533 & 0.1280 & $52.03 \%$ & 1.8543 & 0.1283 & $50.64 \%$ & 1.8323 & 0.1277 \\
\hline
\end{tabular}

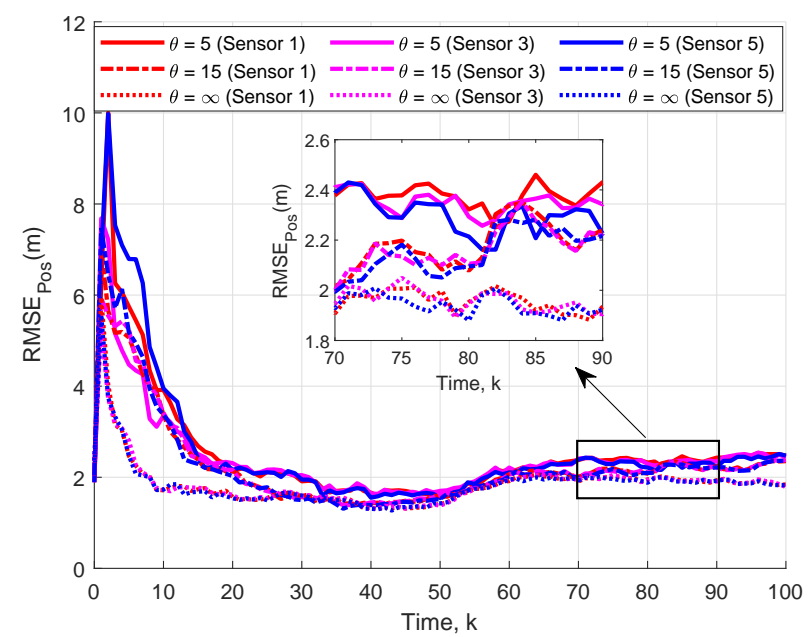

Fig. 9: Position RMSEs of DAPF-DETM with respect to different parameters.

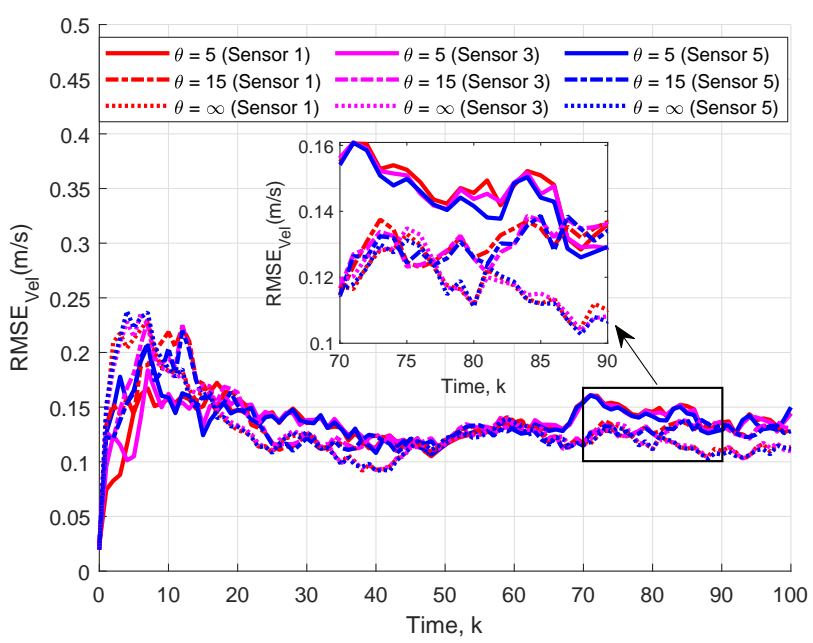

Fig. 10: Velocity RMSEs of DAPF-DETM with respect to different parameters.

the dynamic ETCM has been introduced in both adaptation stage and combination stage, and the local posterior PDF has been represented by the Gaussian mixture model to further save the communication cost. Finally, the proposed algorithms have been applied in the target tracking problem with receivedsignal-strength sensors and compared with other schemes. The simulation results have demonstrated the feasibility and validity of the proposed filtering algorithms. To compress the information contained in the samples into a reduced number of summary samples, it is of great interest to explore the extensions of the present results based on the compressed Monte Carlo scheme [41], the herding algorithm [25] as well as the group importance sampling strategy [42].

\section{REFERENCES}

[1] N. R. Ahmed and M. Campbell, Fast consistent Chernoff fusion of Gaussian mixtures for ad hoc sensor networks, IEEE Transactions on Signal Processing, vol. 60, no. 12, pp. 6739-6745, 2012.

[2] I. Arasaratnam and S. Haykin, Cubature Kalman filters, IEEE Transactions on Automatic Control, vol. 54, no. 6, pp. 1254-1269, 2009.

[3] M. S. Arulampalam, S. Maskell, N. Gordon and T. Clapp, A tutorial on particle filters for online nonlinear/non-Gaussian Bayesian tracking, IEEE Transactions on Signal Processing, vol. 50, no. 2, pp. 174-188, 2002.

[4] X. Bai, Z. Wang, L. Sheng and Z. Wang, Reliable data fusion of hierarchical wireless sensor networks with asynchronous measurement for greenhouse monitoring, IEEE Transactions on Control Systems Technology, vol. 27, no. 3, pp. 1036-1046, May 2019.

[5] G. Battistelli and L. Chisci, Kullback-Leibler average, consensus on probability densities, and distributed state estimation with guaranteed stability, Automatica, vol. 50, no. 3, pp. 707-718, 2014.

[6] G. Battistelli, L. Chisci, L. Gao and D. Selvi, Event-triggered distributed Bayes filter, in Proceedings of the 18th European Control Conference, Naples, Italy, 2019, pp. 2731-2736.

[7] G. Battistelli, L. Chisci and D. Selvi, A distributed Kalman filter with event-triggered communication and guaranteed stability, Automatica, vol. 93, pp. 75-82, 2018.

[8] C. J. Bordin and M. G. S. Bruno, Consensus-based distributed particle filtering algorithms for cooperative blind equalization in receiver networks, in Proceedings of the 2011 IEEE International Conference on Acoustics, Speech and Signal Processing (ICASSP), Prague, 2011, pp. 3968-3971.

[9] M. G. S. Bruno and S. S. Dias, A Bayesian interpretation of distributed diffusion filtering algorithms, IEEE Signal Processing Magazine, vol. 35, no. 3, pp. 118-123, 2018.

[10] M. G. S. Bruno and S. S. Dias, Collaborative emitter tracking using RaoBlackwellized random exchange diffusion particle filtering, EURASIP Journal on Advances in Signal Processing, vol. 19, pp. 1-18, 2014.

[11] F. S. Cattivelli and A. H. Sayed, Diffusion strategies for distributed Kalman filtering and smoothing, IEEE Transactions on Automatic Control, vol. 55, no. 9, pp. 2069-2084, 2010.

[12] Y. Chen, Z. Wang, L. Wang and W. Sheng, Finite-horizon $H_{\infty}$ state estimation for stochastic coupled networks with random inner couplings using Round-Robin protocol, IEEE Transactions on Cybernetics, in press, DOI: $10.1109 /$ TCYB.2020.3004288.

[13] D. Ciuonzo and P. S. Rossi, Distributed detection of a non-cooperative target via generalized locally-optimum approaches, Information Fusion, vol. 36, pp. 261-274, 2017.

[14] D. Ciuonzo, P. S. Rossi and P. Willett, Generalized Rao test for decentralized detection of an uncooperative target, IEEE Signal Processing Letters, vol. 24, no. 5, pp. 678-682, 2017.

[15] M. Coates, Distributed particle filters for sensor networks, in Proceedings of the 3rd International Symposium on Information Processing in Sensor Networks, Berkeley, CA, USA, 2004, pp. 99-107.

[16] S. Davar, A. Mohammadi and K. N. Plataniotis, Event-triggered particle filtering via diffusion strategies for distributed estimation in autonomous systems, in Proceedings of IEEE International Conference on Acoustics, Speech and Signal Processing, Calgary, AB, 2018, pp. 6578-6582.

[17] K. Dedecius and P. M. Djurić, Sequential estimation and diffusion of information over networks: A Bayesian approach with exponential family of distributions, IEEE Transactions on Signal Processing, vol. 65, no. 7, pp. 1795-1809, 2017. 
This article has been accepted for publication in a future issue of this journal, but has not been fully edited. Content may change prior to final publication. Citation information: DOI10.1109/TSP.2020.3042947, IEEE Transactions on Signal Processing

FINAL VERSION

[18] S. S. Dias and M. G. S. Bruno, Cooperative target tracking using decentralized particle filtering and RSS sensors, IEEE Transactions on Signal Processing, vol. 61, no. 14, pp. 3632-3646, 2013.

[19] X. Ge, Q.-L. Han and Z. Wang, A dynamic event-triggered transmission scheme for distributed set-membership estimation over wireless sensor networks," IEEE Transactions on Cybernetics, vol. 49, no. 1, pp. 171$183,2019$.

[20] T. Ghirmai, Distributed particle filter for target tracking: with reduced sensor communications, Sensors (Basel), vol. 16, no. 9, art. no. 1454, 2016.

[21] F. Han, Z. Wang, H. Dong and H. Liu, Partial-nodes-based scalable $H_{\infty}$ consensus filtering with censored measurements over sensor networks, IEEE Transactions on Systems, Man, and Cybernetics-Systems, in press, DOI: 10.1109/TSMC.2019.2907649.

[22] O. Hlinka, F. Hlawatsch and P. M. Djurić, Distributed particle filtering in agent networks: A survey, classification, and comparison, IEEE Signal Processing Magazine, vol. 30, no. 1, pp. 61-81, 2013.

[23] O. Hlinka, F. Hlawatsch and P. M. Djurić, Likelihood consensus-based distributed particle filtering with distributed proposal density adaptation, in Proceedings of the 2012 IEEE International Conference on Acoustics, Speech and Signal Processing (ICASSP), Kyoto, Japan, 2012, pp. 38693872.

[24] J. Hu, L. Xie and C. Zhang, Diffusion Kalman filtering based on covariance intersection, IEEE Transactions on Signal Processing, vol. 60, no. 2, pp. 891-902, 2012.

[25] F. Huszár and D. Duvenaud, Optimally-weighted herding is Bayesian quadrature, in Proceedings of the Twenty-Eighth Conference on Uncertainty in Artificial Intelligence, Catalina Island, CA, 2012, pp. 377-386.

[26] A. H. Jazwinski, Stochastic processing and filtering theory. New York: Academic Press, 1970.

[27] B. Jia, M. Xin and Y. Cheng, Sparse-grid quadrature nonlinear filtering, Automatica, vol. 48, no. 2, pp. 327-341, 2012.

[28] S. Julier, J. Uhlmann and H. F. Durrant-Whyte, A new method for the nonlinear transformation of means and covariances in filters and estimators, IEEE Transactions on Automatic Control, vol. 45, no. 3, pp. 477-482, 2000.

[29] J. Li and A. Nehorai, Distributed particle filtering via optimal fusion of Gaussian mixtures, IEEE Transactions on Signal and Information Processing over Networks, vol. 4, no. 2, pp. 280-292, 2018.

[30] J. Li, Z. Wang, H. Dong and F. Han, Delay-distribution-dependent state estimation for neural networks under stochastic communication protocol with uncertain transition probabilities, Neural Networks, vol. 130, pp. 143-151, Oct. 2020.

[31] Q. Li, B. Shen, Z. Wang and W. Sheng, Recursive distributed filtering over sensor networks on Gilbert-Elliott channels: A dynamic eventtriggered approach, Automatica, vol. 113, art. no. 108681, 9 pages, 2020.

[32] Q. Li, Z. Wang, N. Li and W. Sheng, A dynamic event-triggered approach to recursive filtering for complex networks with switching topologies subject to random sensor failures, IEEE Transactions on Neural Networks and Learning Systems, Vol. 31, No. 10, pp. 4381-4388, Oct. 2020

[33] W. Li and Y. Jia, Distributed estimation for Markov jump systems via diffusion strategies, IEEE Transactions on Aerospace and Electronic Systems, vol. 53, no. 1, pp. 448-460, 2017.

[34] W. Li, Z. Wang, D. W. C. Ho and G. Wei, On boundedness of error covariances for Kalman consensus filtering problems, IEEE Transactions on Automatic Control, in press, DOI: 10.1109/TAC.2019.2942826.

[35] W. Li, Z. Wang, Q. Liu and L. Guo, An information aware eventtriggered scheme for particle filter based remote state estimation, Automatica, vol. 103, pp. 151-158, 2019.

[36] W. Li, Z. Wang, Y. Yuan and L. Guo, Particle filtering with applications in networked systems: a survey, Complex \& Intelligent Systems, vol. 2, no. 4, pp. 293-315, 2016.

[37] D. Liu, Z. Wang, Y. Liu and F. E. Alsaadi, Extended Kalman filtering subject to random transmission delays: Dealing with packet disorders, Information Fusion, vol. 60, pp. 80-86, Aug. 2020.

[38] Q. Liu and Z. Wang, Moving-horizon estimation for linear dynamic networks with binary encoding schemes, IEEE Transactions on Automatic Control, in press, DOI: 10.1109/TAC.2020.2996579.

[39] S. Liu, Z. Wang, Y. Chen and G. Wei, Protocol-based unscented Kalman filtering in the presence of stochastic uncertainties, IEEE Transactions on Automatic Control, vol. 65, no. 3, pp. 1303-1309, Mar. 2020.

[40] L. Ma, Z. Wang, C. Cai and F. E. Alsaadi, Dynamic event-triggered state estimation for discrete-time singularly perturbed systems with distributed time-delays, IEEE Transactions on Systems, Man, and Cybernetics: Systems, in press, DOI: 10.1109/TSMC.2018.2876203.
[41] L. Martino and V. Elvira, Compressed Monte Carlo for distributed Bayesian inference, viXra: 1811.0505, 2018.

[42] L. Martino, V. Elvira and G. Camps-Valls, Group importance sampling for particle filtering and MCMC, Digital Signal Processing, vol. 82, pp. 133-151, 2018.

[43] L. Martino, J. Read, V. Elvira and F. Louzada, Cooperative parallel particle filters for online model selection and applications to urban mobility, Digital Signal Processing, vol. 60, pp. 172-185, 2017.

[44] I. Matei and J. S. Baras, Consensus-based linear distributed filtering, Automatica, vol. 48, no. 8, pp. 1776-1782, 2012.

[45] R. Olfati-Saber, Distributed Kalman filtering for sensor networks, in Proceedings of the 46th IEEE Conference on Decision and Control, New Orleans, LA, 2007, pp. 5492-5498.

[46] N. Patwari, A. O. Hero III, M. Perkins, N. S. Correal and R. J. O'Dea, Relative location estimation in wireless sensor networks, IEEE Transactions on Signal Processing, vol. 51, no. 8, pp. 2137-2148, 2003.

[47] M. Pitt and N. Shephard, Filtering via simulation: Auxiliary particle filters, Journal of the American Statistical Association, vol. 94, no. 446, pp. 590-599, 1999.

[48] J. Read, K. Achutegui and J. Míguez, A distributed particle filter for nonlinear tracking in wireless sensor networks, Signal Processing, vol. 98, pp. 121-134, 2014.

[49] S. Särkkä and A. Nummenmaa, Recursive noise adaptive Kalman filtering by variational Bayesian approximations, IEEE Transactions on Automatic Control, vol. 54, no. 3, pp. 596-600, 2009.

[50] B. Shen, Z. Wang, D. Wang and H. Liu, Distributed state-saturated recursive filtering over sensor networks under Round-Robin protocol, IEEE Transactions on Cybernetics, vol. 50, no. 8, pp. 3605-3615, Aug. 2020.

[51] Y. Shen, Z. Wang, B. Shen and F. E. Alsaadi, $H_{\infty}$ state estimation for multi-rate artificial neural networks with integral measurements: A switched system approach, Information Sciences, vol. 539, pp. 434-446, Oct. 2020.

[52] Y. Shen, Z. Wang, B. Shen, F. E. Alsaadi and F. E. Alsaadi, Fusion estimation for multi-rate linear repetitive processes under weighted Try-Once-Discard protocol, Information Fusion, vol. 55, pp. 281-291, Mar. 2020.

[53] G. M. Siouris, G. Chen and J. Wang, Tracking an incoming ballistic missile using an extended interval Kalman filter, IEEE Transactions on Aerospace and Electronic Systems, vol. 33, no. 1, pp. 232-240, 1997.

[54] J. Suo, Z. Wang and B. Shen, Pinning synchronization control for a class of discrete-time switched stochastic complex networks under eventtriggered mechanism, Nonlinear Analysis: Hybrid Systems, vol. 37, Art. no. 100886, Aug. 2020.

[55] S.-Y. Tu and A. H. Sayed, Diffusion strategies outperform consensus strategies for distributed estimation over adaptive networks, IEEE Transactions on Signal Processing, vol. 60, no. 12, pp. 6217-6234, 2012.

[56] C. Vergé, C. Dubarry, P. D. Moral and E. Moulines, On parallel implementation of sequential Monte Carlo methods: the island particle model, Statistics and Computing, vol. 25, pp. 243-260, 2015.

[57] W. Yu, G. Chen, Z. Wang and W. Yang, Distributed consensus filtering in sensor networks, IEEE Transactions on Systems, Man, and Cybernetics, Part B (Cybernetics), vol. 39, no. 6, pp. 1568-1577, 2009.

[58] L. Xu, K. Ma, W. Li, Y. Liu and F. E. Alsaadi, Particle filtering for networked nonlinear systems subject to random one-step sensor delay and missing measurements, Neurocomputing, vol. 275, pp. 2162-2169, 2018.

[59] Z. R. Zaidi and B. L. Mark, Real-time mobility tracking algorithms for cellular networks based on Kalman filtering, IEEE Transactions on Mobile Computing, vol. 4, no. 2, pp. 195-208, 2005.

[60] Q. Zhang, Z. Chen and F. Yin, Speaker tracking based on distributed particle filter in distributed microphone networks, IEEE Transactions on Systems, Man, and Cybernetics: Systems, vol. 47, no. 9, pp. 2433-2443, 2017.

[61] X.-M. Zhang, Q.-L. Han, X. Ge, D. Ding, L. Ding, D. Yue and C. Peng, Networked control systems: A survey of trends and techniques, IEEECAA Journal of Automatica Sinica, vol. 7, no. 1, pp. 1-17, Jan. 2020.

[62] Y. Zheng, R. Niu and P. K. Varshney, Sequential Bayesian estimation with censored data for multi-sensor systems, IEEE Transactions on Signal Processing, vol. 62, no. 10, pp. 2626-2641, 2014. 
This article has been accepted for publication in a future issue of this journal, but has not been fully edited. Content may change prior to final publication. Citation information: DOI10.1109/TSP.2020.3042947, IEEE Transactions on Signal Processing

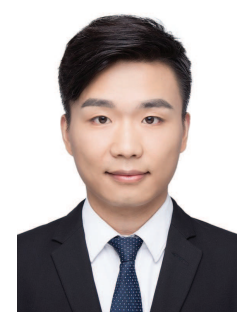

networked control systems.

Weihao Song received the B.S. degree in flight vehicle design and engineering from Beijing Institute of Technology, Beijing, China, in 2016. He is currently pursuing the Ph.D. degree in aeronautical and astronautical science and technology with Beijing Institute of Technology, Beijing, China.

From May 2019 to May 2020, he was a Visiting Scholar with the Department of Computer Science, Brunel University London, London, U.K. His research interests include Bayesian state estimation, distributed state estimation, nonlinear filtering, and

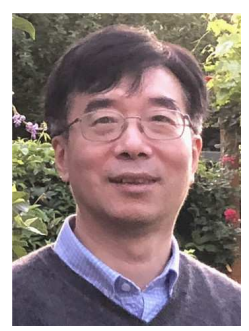

Zidong Wang (SM'03-F'14) was born in Jiangsu, China, in 1966. He received the B.Sc. degree in mathematics in 1986 from Suzhou University, Suzhou, China, and the M.Sc. degree in applied mathematics in 1990 and the Ph.D. degree in electrical engineering in 1994, both from Nanjing University of Science and Technology, Nanjing, China.

$\mathrm{He}$ is currently a Professor of Dynamical Systems and Computing in the Department of Computer Science, Brunel University London, U.K. From 1990 to 2002, he held teaching and research appointments in universities in China, Germany and the UK. Prof. Wang's research interests include dynamical systems, signal processing, bioinformatics, control theory and applications. He has published around 600 papers in refereed international journals. He is a holder of the Alexander von Humboldt Research Fellowship of Germany, the JSPS Research Fellowship of Japan, William Mong Visiting Research Fellowship of Hong Kong.

Prof. Wang serves (or has served) as the Editor-in-Chief for Neurocomputing, Deputy Editor-in-Chief for International Journal of Systems Science, and an Associate Editor for 12 international journals including IEEE Transactions on Automatic Control, IEEE Transactions on Control Systems Technology, IEEE Transactions on Neural Networks, IEEE Transactions on Signal Processing, and IEEE Transactions on Systems, Man, and Cybernetics-Part C. $\mathrm{He}$ is a Member of the Academia Europaea, a Fellow of the IEEE, a Fellow of the Royal Statistical Society and a member of program committee for many international conferences.

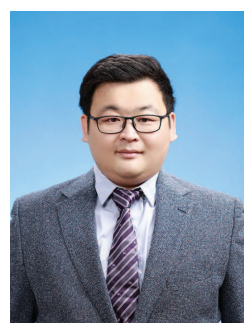

Jianan Wang (Senior Member, IEEE) received the B.S. and M.S. degrees in control theory and engineering from Beijing Jiaotong University and Beijing Institute of Technology, Beijing, China, in 2004 and 2007, respectively. He received the Ph.D. degree in aerospace engineering from Mississippi State University, Starkville, MS, USA, in 2011.

$\mathrm{He}$ is currently an Associate Professor with the School of Aerospace Engineering, Beijing Institute of Technology. His research interests include cooperative control of multiple dynamic systems, UAV formation control, cooperative guidance, and estimation of sensor networks. $\mathrm{He}$ is a senior member of IEEE and AIAA.

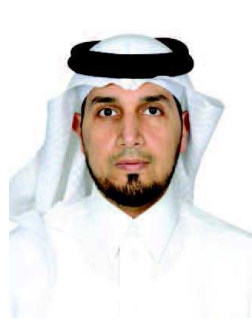

Fuad E. Alsaadi received the B.S. and M.Sc. degrees in electronic and communication from King AbdulAziz University, Jeddah, Saudi Arabia, in 1996 and 2002. He then received the Ph.D. degree in Optical Wireless Communication Systems from the University of Leeds, Leeds, UK, in 2011. Between 1996 and 2005, he worked in Jeddah as a communication instructor in the College of Electronics \& Communication. He is currently an associate professor of the Electrical and Computer Engineering Department within the Faculty of Engineering, King Abdulaziz University, Jeddah, Saudi Arabia. He published widely in the top IEEE communications conferences and journals and has received the Carter award, University of Leeds for the best PhD. He has research interests in optical systems and networks, signal processing, synchronization and systems design.

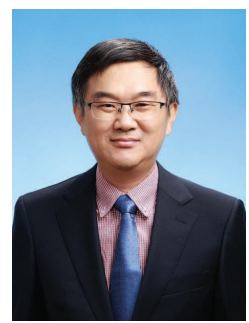

Jiayuan Shan received the B.S. degree from Huazhong University of Science and Technology, Wuhan, China, in 1988, and the M.S. and Ph.D. degrees from Beijing Institute of Technology, Beijing, China, in 1991 and 1999, respectively.

$\mathrm{He}$ is currently a Professor with the School of Aerospace Engineering, Beijing Institute of Technology. His research interests include guidance, navigation and control of the aircraft and hardware-in-theloop simulation. He is the Principal Professor in the direction of Flight Dynamics and Control. 